Doi: $\underline{\mathrm{dx} . \text { doi.org/10.17921/2525-5320.2016.74-84 }}$

\title{
DOCÊNCIA E MOTIVAÇÃO PARA LEITURA: UM ESTUDO COM ADOLESCENTES E A PERCEPÇÃO DE SEUS PAIS
}

Maria Fernanda Cunha Oliveira* - UEL

Palavras-chave: Docência. Motivação para Leitura. Adolescente.

\section{INTRODUÇÃO}

Cada vez há mais necessidade de prestar atenção nas angústias do professor para poder responder com eficácia aos desafios do atual sistema educativo. Nesta direção, percebe-se que uma das principais queixas dos professores de diversas disciplinas do currículo escolar, em todos os níveis da escolaridade, é relativa à falta de competência de seus alunos para interpretarem textos o que é justamente atribuível, em boa parte, à baixa frequência de leitura. O ato de ler é um processo altamente complexo que vai muito além da simples decodificação, haja vista envolver o reconhecimento de letras e palavras, compreensão de significados, capacidade de inferir, memória, conhecimento de mundo, dentre outros. De acordo com Witter (2010), ler é um comportamento que pode ser aprendido e melhorado durante a vida, a autora argumentou, porém, que o ato de ler depende de motivação para acontecer qualitativamente e se manter.

A motivação tornou-se um problema de ponta em educação e tem sido definida como "um processo por meio do qual atividades direcionadas a metas são instigadas e sustentadas" (SCHUNK; MEECE; PINTRICH, 2014, p.5). Estes autores entendem a motivação como um processo, em vez de um produto e, como tal, envolve a presença de ações, como, por exemplo, escolha, esforço, persistência, metas, planejamento, organização, monitoramento, tomada de decisões, resolução de problemas e avaliação do processo. Além disso, pesquisadores, a exemplo de Wigfield (1997), Wigfield, e Guthrie (1997), desenvolveram estudos na área da motivação para ler e identificaram queda na motivação dos estudantes com o passar dos anos escolares. Sendo assim, pode-se inferir que os alunos concebem o ensino pouco significativo e se encontram divididos entre a necessidade de prosseguir, pois

*E-mail: mfernanda_cunha@yahoo.com.br 
o estudo é obrigatório e há falta de motivação.

A motivação do aluno pode variar por disciplina em função das diferentes exigências e dificuldade de cada uma, grau de interesse, valorização e autopercepção de capacidade. Neste contexto, além de ser um processo específico, a motivação para a leitura tem sido vista pelos pesquisadores como um constructo multidimensional, assim como a própria motivação do aluno na escola. Isto é, nessa motivação específica atuam diversos fatores, tendo-se identificado, segundo Wigfield (1997), Guthrie e Wigfield (2000), constructos como: (a) as crenças do indivíduo sobre sua competência e expectativas de eficácia para ler; (b) percepções de interesse, importância e utilidade de ler (valorização subjetiva da tarefa); (c) curiosidade por leitura como um fim em si mesmo (motivação intrínseca); e (d) adoção da meta domínio, não de performance.

Portanto, a motivação é necessária quando se trata da aquisição de habilidades de leitura visto que estudos comprovaram uma estreita ligação entre hábitos de leitura e compreensão de textos, pois se identificou que o baixo nível de compreensão está correlacionado à falta de leitura causada por desmotivação. Sendo assim, a motivação é crucial para que tais atividades aconteçam.

Embora o valor e a relevância da leitura, seja na escola ou em outros contextos, tenha sido documentada na literatura (BAPTISTA et al., 2016; LAW, 2011), praticar leitura de livros e textos, além dos livros didáticos básicos, exige uma motivação sustentada e de qualidade, visto que compete com atividades mais valorizadas pelos adolescentes, como estar com os amigos e entreter-se com equipamentos eletrônicos.

Em síntese, com base nos relatos dessa amostra de estudos, conclui-se que a motivação para leitura é constructo multidimensional o qual aparece em graus diversos entre os alunos, com diferenças entre os sexos, e tende a diminuir à medida que progridem nos anos escolares. Por este motivo, vale ressaltar a importância de pesquisas na área, envolvendo alunos do final do Ensino Fundamental II e Ensino Médio.

Outro fator relevante é que o desempenho dos adolescentes e a motivação para qualquer uma das atividades de aprendizagem são positivamente influenciados pela família. A literatura tem fornecido evidências sobre a relação entre determinados comportamentos dos pais e a valorização da leitura por parte dos seus 
filhos. Witter (2010) enfatizou a importância de hábitos de leitura familiares, o que faz com que os pais sejam vistos como modelos de apreciação da função social da leitura. Na mesma direção, variáveis como qualificação dos pais e o número de livros em casa, regularmente utilizados, foram relacionados positivamente com frequência de comunicação pais-filhos sobre assuntos escolares e com desempenho na escola.

Para fundamentar teoricamente este estudo, elegeu-se a Teoria da Autodeterminção, pois, partindo do pressuposto de que existem pessoas motivadas intrinsicamente e motivadas extrinsecamente, a teoria busca entender e explicar estas formas motivacionais e relacioná-las com as condições ambientais que podem nutrir ou minar a motivação dos alunos (DECl; RYAN, 2008; RYAN; DECl, 2004). A Teoria da Autodeterminação - TAD foi elaborada por Deci, Ryan e colaboradores entre os anos 1970 e 1980. Tais autores consideram a motivação, em termos das razões para agir, sendo um conceito multidimensional que varia em termos de qualidade, em função de processos de regulação (DECl, RYAN, 1985; 2000; RYAN, DECl, 2004). Nesta perspectiva, existem comportamentos mais ou menos regulados, com significados motivacionais bem distintos, os quais variam de acordo com o continuum da autorregulação. Por primeiro, destaca-se a ocorrência da desmotivação, seguida do desmembramento da motivação extrínseca em quatro níveis de regulação, a saber: regulação externa, regulação introjetada, regulação identificada e a regulação integrada. A motivação intrínseca vem na sequência e representa a forma mais autodeterminada de regulação, haja vista que se caracteriza pelo interesse e prazer no exercício de uma atividade.

Portanto, entender os diferentes níveis de motivação extrínseca é fundamental para o educador, pois o mesmo não pode confiar plenamente que apenas a motivação intrínseca promove um aprendizado significativo. Considerando que nem todas as tarefas escolares são interessantes e agradáveis, saber como promover formas mais ativas e volitivas de motivação extrínseca pode ser uma estratégia valiosa para o ensino bem-sucedido (RYAN; DECl, 2000).

Ainda neste contexto, é relevante destacar que, como Deci e Ryan (2000) defenderam que o ponto mais crítico na teoria é a distinção entre motivação autônoma e controlada. Assim, a motivação autônoma compreende a motivação intrínseca e as duas formas autorreguladas da motivação extrínseca, a identificada e a integrada. Já as motivações por regulação extrínseca e introjetada formam a 
motivação controlada. Porém, segundo Ratelle (2007) a motivação autônoma e a motivação controlada não necessitam ser polos opostos, elas podem relacionar-se entre si.

Nesta direção, a abordagem centrada no aluno (Person-Oriented Approach) foi sugerida por Ratelle et al. (2007) que, apoiados em Pintrich (2003), propuseram a combinação dos escores em mais de uma escala. Por exemplo, um aluno pode realizar as atividades de leitura por se identificar com o livro escolhido pelo professor e, ao mesmo tempo porque não aceita tirar notas baixas. Assim, como o foco é no aluno e não nas variáveis, esta abordagem permite a identificação de grupos homogêneos (clusters) que compartilham determinadas características. Desta maneira, os alunos podem ser agrupados em clusters, por exemplo, com altos escores em motivação autônoma e baixos em motivação controlada; altos escores em motivação controlada e baixos em motivação autônoma; altos escores em motivação autônoma e altos em motivação controlada ou ainda, baixos escores em motivação autônoma e baixos em motivação controlada. Ratelle (2007) ainda argumentou que os estudos que utilizam a abordagem centrada no aluno têm resultados mais positivos, pois, analisam como diferentes níveis de motivação se combinam e formam distintos perfis motivacionais.

Este método foi escolhido para o tratamento de dados desta pesquisa e também foi utilizado por outros estudiosos da TAD (BOICHÉ et al., 2008; BZUNECK; MEGLIATO; RUFINI 2013; RATELLE et al., 2007, VANSTEENKISTE et al., 2009; WORMINGTON et al., 2012).

No presente estudo, buscou-se examinar a motivação para leitura de uma amostra de estudantes adolescentes e partiu do seguinte questionamento: Por quais razões e em que grau os alunos se dedicam à leitura de livros prescritos pelos seus professores, afora os textos didáticos? Apenas por que são obrigados? E qual a influência dos pais para a valorização da leitura? Assim, com a intenção de ampliar o conhecimento baseado em pesquisas brasileiras sobre o tema, ainda escassas, buscou-se identificar neste estudo perfis motivacionais de alunos adolescentes para a leitura, além de relacionar cada perfil motivacional com a percepção de comportamentos dos pais ligados a essa área.

Mais especificamente, foi propósito deste estudo investigar (1) a qualidade motivacional de adolescentes para a leitura, identificados por clusters ou perfis que 
combinem motivação autônoma e controlada;

(2) em que proporção esses adolescentes se localizam em cada cluster, quando discriminados por gênero e pelo tipo de escola que frequentam, pública ou particular; e (3) a relação entre percepção de comportamentos dos pais acerca de leitura e o perfil de cada cluster, como indicador da qualidade motivacional dos alunos.

\section{MATERIAL E MÉTODOS}

\section{Participantes}

A pesquisa foi realizada com 566 adolescentes do $9^{\circ}$ ano do Ensino Fundamental II e do $2^{\circ}$ ano do Ensino Médio de duas escolas públicas ( $n=392$, ou $69,2 \%$ ) e duas particulares $(n=174$, ou 30,8\%) do interior do Paraná. De toda a amostra, contavam-se 257 (45,5\%) do sexo masculino, 308 (54,5\%) do sexo feminino e uma omissão do dado.

A Figura 1 mostra a distribuição dos participantes concomitantemente nos três fatores. Ou seja, na motivação autônoma e controlada e na percepção sobre os pais.

Figura 1: Figura de caixas da distribuição dos valores nas diversas subescalas: Fator 1 (Mot. intrínseca); Fator 2 (Mot. por Regulação Externa; e Fator 3 (Mot. Identificada)

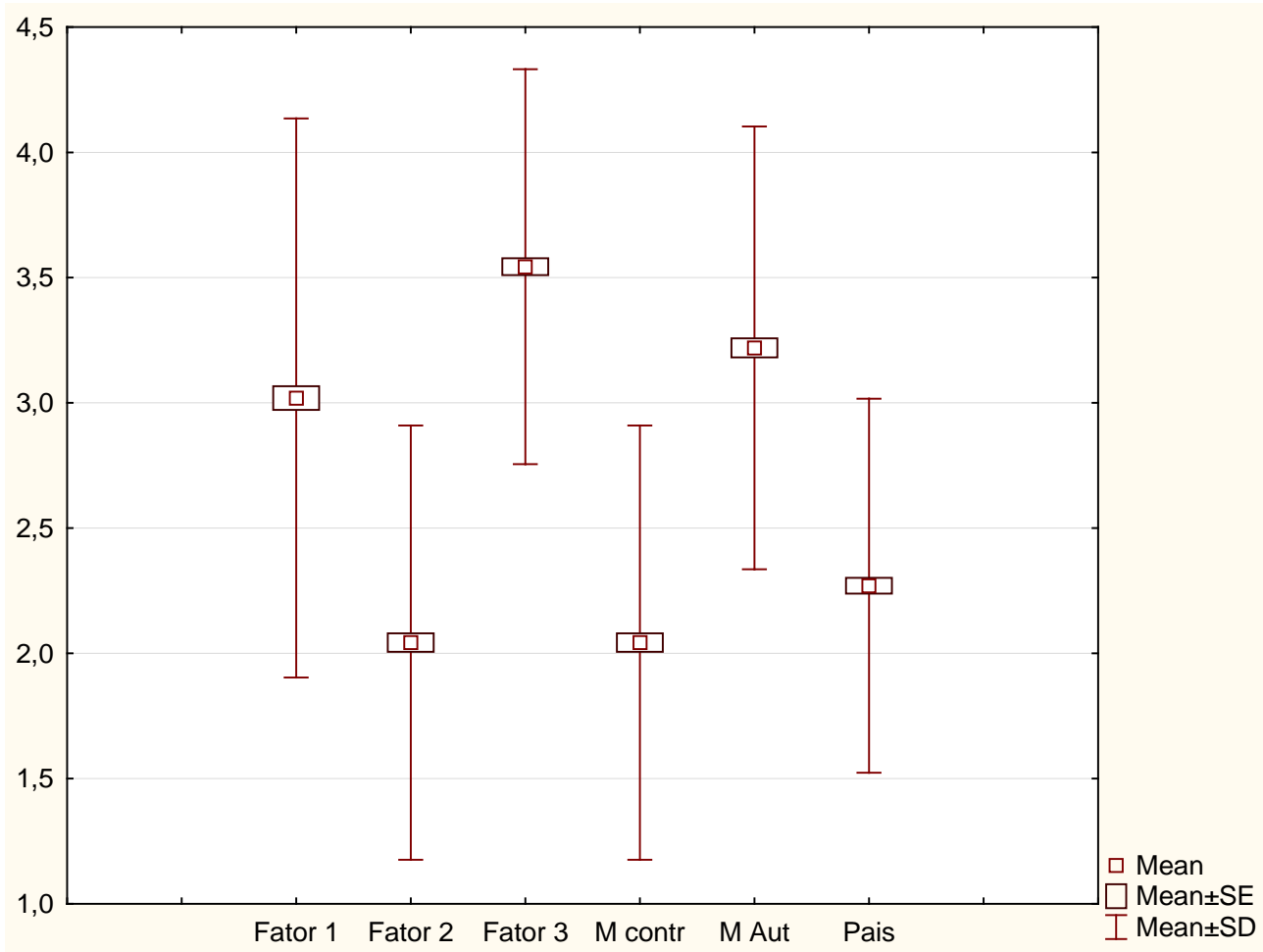

Fonte: O autor 
Um questionário de autorrelato denominado "Inventário sobre Motivação para Leitura", com 28 questões fechadas em escala tipo Likert relativas à motivação para a leitura de livros obrigatórios durante o ano letivo. O mesmo levantou informações a respeito a) da motivação intrínseca; b) da motivação por regulação externa; c) da motivação identificada e d) percepção de pais.

Nesta investigação, atenderam-se às exigências constantes da Resolução n466/2012 do Conselho Nacional de Saúde e, para tanto, o projeto foi submetido, no devido tempo, ao Comitê de Ética em Pesquisa da Universidade Estadual de Londrina e aprovado sob o parecer $n^{\circ}$ 155/2014.

\section{RESULTADOS}

No Quadro figuram, para a amostra total de alunos, dados descritivos relativos às três variáveis motivacionais correspondentes aos três fatores, à motivação autônoma e à controlada e, ainda, à percepção de pais.

Quadro 1: Estatística descritiva das variáveis e valores de correlação de Pearson em relação à amostra total $(n=556)$

\begin{tabular}{|c|c|c|c|c|c|c|c|c|c|c|}
\hline Variável & $M$ & D P & Assim. & Curtose & 1 & 2 & 3 & 4 & 5 & 6 \\
\hline 1. Motivação Intríns. & 3,01 & 1,11 & 0,01 & $-1,13$ & - & $-0,59$ & 0,49 & $-0,59$ & 0,95 & 0,30 \\
\hline 2.Regulação Externa & 2,04 & 0,86 & 0,86 & $-0,13$ & - & - & \multicolumn{2}{|c|}{$-0,191,00$} & $-0,53$ & $-0,12$ \\
\hline 3.Regulação Identif. & 3,54 & 0,78 & $-0,45$ & $-0,04$ & - & - & - & $-0,19$ & 0,72 & 0,19 \\
\hline $\begin{array}{l}\text { 4.Motivação Autôn. } \\
\text { 5.Motivação Contr. } \\
\text { 6.Percepção Pais }\end{array}$ & $\begin{array}{l}3,21 \\
2,04 \\
2,27\end{array}$ & $\begin{array}{l}0,88 \\
0,86 \\
0,74\end{array}$ & $\begin{array}{l}-0,16 \\
0,86 \\
0,20\end{array}$ & $\begin{array}{l}-0,80 \\
-0,13 \\
-0,75\end{array}$ & $\begin{array}{l}- \\
- \\
-\end{array}$ & $\begin{array}{l}- \\
- \\
-\end{array}$ & $\begin{array}{l}- \\
- \\
-\end{array}$ & $\begin{array}{l}- \\
- \\
-\end{array}$ & $\begin{array}{c}-0,53 \\
- \\
-\end{array}$ & $\begin{array}{r}0,30 \\
-0,12 \\
-\end{array}$ \\
\hline
\end{tabular}

Nota: Todas as correlações são significativas $(p=0,01)$

Fonte: $\mathrm{O}$ autor.

Com base nesses dados descritivos preliminares, formaram-se, pelo método $k$ means, os perfis motivacionais ou clusters de alunos em função dos escores combinados de motivação autônoma e controlada.

A Tabela 2 mostra a distribuição dos alunos nos dois clusters, discriminados por sexo e tipo de escola, particular ou pública.

Quadro 2: Distribuição dos alunos nos dois clusters, discriminados por sexo e tipo de escola, particular ou pública

\begin{tabular}{l|l} 
Masc & Fem
\end{tabular}

\begin{tabular}{l|l|} 
Fem & Total
\end{tabular}

Part

Publ Total 


\begin{tabular}{|c|c|c|c|c|c|c|c|c|c|c|}
\hline & $\mathbf{n}$ & $\mathbf{\%}$ & $\mathbf{n}$ & $\mathbf{\%}$ & $\mathbf{n}$ & $\mathbf{n}$ & $\mathbf{\%}$ & $\mathbf{n}$ & $\mathbf{\%}$ & $\mathbf{n}$ \\
\hline Cluster 1 & 84 & 36,4 & 184 & 63,7 & 268 & 72 & 45 & 196 & 54,4 & 268 \\
\hline Cluster 2 & 147 & 63,6 & 105 & 36,3 & 252 & 88 & 55 & 164 & 45,5 & 252 \\
\hline Total & 231 & 100 & 289 & 100 & 520 & 160 & 100 & 360 & 100 & 520 \\
\hline
\end{tabular}

Fonte: Dados da pesquisa.

De acordo com Ratelle et al. (2007), a motivação autônoma e a controlada não são, necessariamente, dois polos opostos, mas, ao contrário, podem vir combinadas em uma mesma pessoa. Por exemplo, um aluno pode realizar as atividades de leitura por se identificar com o livro escolhido pelo professor e, ao mesmo tempo, porque não aceita tirar notas baixas.

Assim, o aluno pode buscar atividades que the proporcionem prazer e, concomitantemente, estar atento às consequências extrínsecas dessas. Neste contexto, em que há prevalência de recompensas e punições, é crucial que o professor esteja mais sensível às necessidades dos alunos e seja promotor de autonomia, para que os alunos se desenvolvam e tornem-se cada vez mais autônomos.

A questão essencial neste estudo foi, no entanto, investigar como esses alunos se relacionam qualitativamente dentro dos perfis motivacionais. Um olhar mais refinado de diferenças entre combinações únicas de comportamentos motivacionais permite uma abordagem centrada na pessoa. Por meio da identificação de dois diferentes perfis motivacionais, usando análise de cluster, o atual estudo pode delinear mais profunda e especificamente como diferentes grupos de alunos se diferenciam quanto às razões para realizar atividades de leitura. Por isso, neste estudo, adotou-se essa abordagem como proposta de análise dos dados, pois, conforme argumentação de Ratelle (2007), os estudos que a utilizam apresentam resultados mais positivos.

Quanto à distribuição dos perfis por sexo, pode-se inferir que, para esta amostra, as meninas apresentam motivação de melhor qualidade para leitura. Vale ressaltar que o fato de as meninas estarem mais representadas no cluster 1 é uma tendência estatística e este resultado está alinhado com a literatura, a qual mostra que elas apresentam níveis mais altos de motivação para leitura que os meninos (GUAY et al., 2010; MATA et al., 2009; WIGFIELD; GUTHRIE, 1997).

Em relação às escolas, os dados revelaram que as escolas públicas estão melhores representadas no cluster 1 (motivação de boa qualidade) e as escolas 
particulares estão melhores representadas no cluster 2 (motivação moderada). Pode-se inferir que os alunos das escolas públicas são menos cobrados em relação à leitura e este fato pode torná-los mais autônomos em suas escolhas. Portanto, como as escolas públicas deste estudo não tinham um método sistemático de cobrança, seus alunos alegam ser mais autodeterminados.

No entanto, vale ressaltar que, de acordo com a Teoria da Integração Organísmica, uma miniteoria da Teoria da Autodeterminação, a motivação extrínseca pode ser alterada e se tornar autodeterminada, ocorrendo em um continuum de autodeterminação, que vai da desmotivação à motivação intrínseca. Neste contexto, a cobrança das escolas particulares é muito valiosa, pois, não se pode esperar que todos os adolescentes gostem de ler, principalmente nos dias atuais em que a tecnologia é extremamente sedutora. Assim, o papel da escola é fazer com que os alunos passem pelo processo de interiorização e integração, retirando aos poucos a cobrança e as recompensas externas para que seus alunos apresentem, cada vez mais, autonomia em suas ações (RYAN; DECl, 2000).

As análises feitas para verificar a relação entre a percepção sobre os pais e os clusters, como citado anteriormente, os pais precisam mostrar a seus filhos que valorizam e apreciam as atividades de leitura, sendo referência para estes adolescentes. Nesta direção, Dearing et al. (2006) concluíram, com base em sua revisão de literatura, que o envolvimento dos pais com seus filhos na escola tem efeitos sobre os processos de socialização para o desempenho mediante reforçamento e modelação. Isto significa que, de modo geral, os pais que aparecem como modelos de valorização da leitura e que sabem reforçar comportamentos ligados a tais atividades têm filhos mais autônomos e com melhor rendimento escolar. Da mesma forma, Villiger et al. (2012) contribuíram argumentando que a colaboração dos pais com a escola nos projetos de intervenção para promover motivação para leitura e compreensão leitora produziu nas crianças mais satisfação e curiosidade por leitura. E, ainda em relação à importância dos pais serem modelos nas atividades de leitura para seus filhos, é importante ressaltar que, conforme a teoria da autodeterminação (Ryan e Deci, 2000), as formas mais autônomas da motivação em sentido qualitativo ocorrem por interiorização do valor ou importância das aprendizagens, demonstradas por pessoas significativas como, por exemplo, os pais. 


\section{CONCLUSÃO}

Os resultados encontrados neste estudo estão alinhados com resultados anteriores de pesquisas internacionais. Todavia, são novos na medida em que retratam os perfis motivacionais e a percepção sobre os pais. Em síntese, os mesmos representam um avanço nas pesquisas em motivação no Brasil, sobretudo no que tange à motivação para leitura.

Conclui-se este estudo ressaltando uma vez mais a importância de se estudar a motivação para a leitura dos adolescentes, mais especificamente no último ano do Ensino Fundamental II e no Ensino Médio, por meio de uma abordagem centrada na pessoa do aluno. Pela investigação de como os tipos de motivação se combinam, identificaram-se grupos homogêneos (clusters) que compartilharam determinadas características. Este fato evita o reducionismo de conceituar a docência apenas como as atividades que os professores realizam em sala de aula e permite aos mesmos um conhecimento mais refinado da turma, possibilitando o planejamento de atividades de leitura relevantes para cada perfil. Por outro lado, percebe-se a importância da valorização dos pais em relação à leitura de seus filhos. Além disso, este estudo fornece suporte para a perspectiva qualitativa da Teoria da Autodeterminação.

\section{REFERÊNCIAS}

BAPTISTA, R.M. et al. Práticas de leitura e compreensão de texto no $6^{\circ}$ e $7^{\circ}$ anos do ensino fundamental. Estud. Psicol., v.33, n.1, p.173-182, 2016.

BZUNECK, J.A. A motivação do aluno: aspectos introdutórios. In: BORUCHOVITCH, E.; BZUNECK, J.A. (Org.). A motivação do aluno: contribuições da psicologia contemporânea. Petrópolis: Vozes, 2009. p.9-36.

BZUNECK, J.A. Como motivar os alunos: sugestões práticas. In: BORUCHOVITCH, E.; BZUNECK, J.A.; GUIMARÃES, S.E.R. (Org.). Motivação para aprender: aplicações no contexto educativo. Petrópolis: Vozes, 2010. p.13-42.

BZUNECK, J.A.; GUIMARÃES, S.É.R. A promoção da autonomia como estratégia motivacional na escola: uma análise teórica e empírica. In: BZUNECK, J.A.; GUIMARÃES, S.E.R. (Org.). Motivação para aprender: aplicações no contexto educativo. Petrópolis: Vozes, 2010. p.43-70.

BZUNECK, J.A.; GUIMARÃES, S.É.R. Estilos de Professores na Promoção da Motivação Intrínseca: Reformulação e validação de instrumento. Psicol. Teoria Pesq., v.23, n.4, p.415-422, 2007. 
BZUNECK, J.A.; MEGLIATO, J.G.P.; RUFINI, S.É. Engajamento de adolescentes nas tarefas escolares de casa: uma abordagem centrada na pessoa. Psicol. Escolar Educ., v.17, p.151-161, 2013.

BOICHE, J.C.S. et al. Students' motivational profiles and achievement outcomes in physical education: a self-determination perspective. J. Educ. Psychol., v.100, n.3, 688-701, 2008.

GUTHRIE, J.T.; WIGFIELD, A. Engagement and motivation in Reading. In: KAMIL et al. Handbook of reading research. Mahwah: Erlbaum, 2000. p. 403-422

DECI, E.L.; RYAN, R.M. Intrinsic motivation and self-determination in human behavior. New York: Plenum, 1985.

DECI, E.L.; RYAN, R.M. A motivational approach to self: Integration in personality. In: DIENSTBIER, R. (Nebraska symposium on motivation: perspectives on motivation, $v$. 38. p. 237-288.1991.

DECI, E.L.; RYAN, R.M. The "what" and "why" of goal pursuits: human needs and self-determination of behavior. Psychological Inquiry, v.11, n.4, p.227-268, 2000.

DECI, E.L.; RYAN, R.M. Facilitating optimal motivation and psychological well- being across life's domains. Canadian Psychol., v.49, n.1, p.14-23, 2008.

DUMONT, $H$. et al. Does parental homework involvement mediate the relationship between family background and educational outcomes? Contempory Psychol., v.37, p.55-69, 2012.

GARCIA, C.M. Formação de professores para uma mudança educativa. São Paulo: Porto, 2013.

GUAY, F.; RATELLE, C. F.; CHANAL, J. Optimal Learning in Optimal Contexts: The Role of Self-Determination in Education. Canadian Psychol., v.49, n.3, p.233-240, 2008.

GUAY, F.; VALLERAND, R.J.; BLANCHARD, C. On the assessment of situational intrinsic and extrinsic motivation: the situational motivation scale (SIMS). Motivation Emotion, v. 24, n.3, p.175-213, 2000.

GUAY, F. et al. Intrinsic, identified, and controlled types of motivation for school subjects in Young elementary school children. British J. Educ. Psychol., v.80, p.711735, 2010.

KOSKINEN, P.S. et al. Book access, shared reading, and audio models: The effects of supporting the literacy learning of linguistically diverse students in school and at home. J. Educ. Psychol., v. 92, n. 1, p. 23-36, 2000.

LAW, Y-K. The role of teacher's cognitive support in motivating young Hong Kong 
Chinese children to read and enhancing reading comprehension. Teaching Teacher Education, v.27, p.73-84, 2011.

MATA, L.; MONTEIRO, V.; PEIXOTO, F. Motivação para leitura ao longo da escolaridade. Análise Psicol., v.4, p.563-572, 2009.

RATELLE, C.F.; GUAY, F.; VALLERAND, R.J. Autonomous, controlled, and amotivated types of academic motivation: a person-oriented analysis. J. Educ. Psychol., v. 99, n.4, p.734-746, 2007.

RETELSDORF, J.; KÖLLER, O.; MÖLLER. On the effects od motivation on reading performance growth in the secondary school. Learning Instruction, n.21, p.550-559, 2011.

RUFINI, S.E.; BZUNECK, J.A.; OLIVEIRA, K.L. Estudo de validação de uma medida de avaliação da motivação para alunos do ensino fundamental. Psico-USF, v.16, n.1, p.1-9, 2011.

RUFINI, S.E.; BZUNECK, J.A.; OLIVEIRA, K.L. A Qualidade da motivação em estudantes do ensino fundamental. Psico-USF, v.22, n.51, p.53-62, 2012.

WIGFIELD, A. Reading motivation: a domain-specific approach to motivation. Educ. Psychol., v.32, n.2, p.59-68, 1997.

WIGFIELD, A.; GUTHRIE, J. Relations of children's motivation for reading to the amount and breadth of their reading. J. Educ. Psychol., v.89, p.420-432, 1997.

WITTER, G.P. Motivação e leitura. In: BZUNECK, J.A.; GUIMARÃES, S.E.R. (Org.). Motivação para aprender: aplicações no contexto educativo. Petrópolis: Vozes, 2010. p.169-192.

SCHUNK, D.H.; MEECE, J.L.; PINTRICH, P.R. Motivation in education: theory, research and applications. Boston: Pearson, 2014. 